\title{
ON A DENSITY PROBLEM FOR ELLIPTIC CURVES OVER FINITE FIELDS*
}

\author{
YEN-MEI J. CHEN ${ }^{\dagger}$ AND JING YU ${ }^{\ddagger}$
}

\begin{abstract}
We prove an analogue of Artin's primitive root conjecture for two-dimensional tori $\operatorname{Res}_{K / \mathbb{Q}} \mathbb{G}_{m}$ under the Generalized Riemann Hypothesis, where $K$ is an imaginary quadratic field. As a consequence, we are able to derive a precise density formula for a given elliptic curve $E$ over a finite prime field. One adjoins coordinates of all $\ell$-torsion points to the base field and asks for the density of the rational primes $\ell$ for which the resulting Galois extension over the base field has degree $\ell^{2}-1$. It turns out that the density in question is essentially independent of the curves, and unless in certain special cases, even independent of the characteristic $p$ if $p \not \equiv 1(\bmod 4)$.
\end{abstract}

1. Introduction. Given an elliptic curve $E_{/ \mathbb{F}_{p}}$, one is interested in the Galois representations on $\ell$-torsion $E[\ell] \subset E\left(\bar{F}_{p}\right)$ for various rational prime numbers $\ell$. Let $\mathbb{F}_{p}(E[\ell])$ be the Galois extension of $\mathbb{F}_{p}$ obtained by adjoining all coordinates of points in $E[\ell]$. A basic question is: how often the degree $\left[\mathbb{F}_{p}(E[\ell]): \mathbb{F}_{p}\right]$ can be the largest possible, in other words, is equal to $\ell^{2}-1$ ?

If the given curve $E_{/ \mathbb{F}_{p}}$ is supersingular, it is not difficult to deduce that for almost all $\ell$, the degree of $\mathbb{F}_{p}(E[\ell]) / \mathbb{F}_{p}$ is bounded by $2(\ell-1)$. Thus for our purpose it suffices to consider non-supersingular elliptic curves. We want to study the following set associated to a given non-supersingular $E_{/ \mathbb{F}_{p}}$ :

$$
M_{E}=\left\{\ell: \ell \text { rational prime, }\left[\mathbb{F}_{p}(E[\ell]): \mathbb{F}_{p}\right]=\ell^{2}-1\right\}
$$

Our main result is that, under the generalized Riemann Hypothesis (GRH), these sets $M_{E}$ always have positive density. Furthermore the value of this density $\operatorname{den}\left(M_{E}\right)$ can be given precisely in terms of a universal constant $C_{2}$ :

$$
C_{2}=\frac{1}{4} \prod_{q \neq 2}\left(1-\frac{2}{q(q-1)}\right)=0.133776 \cdots
$$

If $p \not \equiv 1(\bmod 4)$, then always $\operatorname{den}\left(M_{E}\right)=C_{2}$ unless in certain exceptional cases. Otherwise we have $\operatorname{den}\left(M_{E}\right)=\left(1-\frac{2}{p(p-1)}\right)^{-1} C_{2}$ (c.f. Theorem 4.3).

The approach of this paper is based on a variation of Artin's primitive root problem for a family of two-dimensional tori over $\mathbb{Q}$. Let $\operatorname{End}_{E}$ denote the endomorphism ring of the elliptic curve $E$ and let $\alpha \in \operatorname{End}_{E}$ be the Frobenius endomorphism. If $E$ is not supersingular, $\mathbb{Z}[\alpha] \subset \operatorname{End}_{E}$, and $\mathbb{Z}[\alpha]$ is identified with an order in an imaginary quadratic field $K=K_{E}$. Then $\mathbb{Z}[\alpha] \subset \mathcal{O}_{K}$, the ring of integers in $K$. The torus in question is the one obtained from $\mathbb{G}_{m / K}$ via restriction of scalars : $\mathbb{T}=\operatorname{Res}_{K / \mathbb{Q}} \mathbb{G}_{m / K}$. This $\mathbb{T}$ is a two-dimensional torus defined over $\mathbb{Q}$. It comes with a canonical homomorphism $\pi: \mathbb{T} \rightarrow \mathbb{G}_{m}$ defined over $K$ which is universal, in the sense that any map into $\mathbb{G}_{m}$ defined over $K$ can be factored through a map into $\mathbb{T}$ that is defined over $\mathbb{Q}$. One identifies $\mathbb{T}(\mathbb{Q})$ with $\mathbb{G}_{m}(K)=K^{\star}$. Therefore the Frobenius endomorphism $\alpha$ is regarded here as a rational point in $\mathbb{T}(\mathbb{Q})$. One observes that powers of such a

* Received June 20, 2000; accepted for publication October 5, 2000. Research partially supported by National Science Council, Rep. of China.

† Department of Mathematics, Tamkang University, Tamshui, Taipei, Taiwan (ymjchen@mail. tku.edu.tw).

$\ddagger$ Institute of Mathematics, Academia Sinica, Nankang, Taipei, Taiwan and National Center for Theoretical Sciences, Hsinchu, Taiwan (yu@math.sinica.edu.tw). 
point can never be contained in any proper subtorus of $\mathbb{T}$. Hence it has a good chance to become "primitive" when reducing modulo rational primes $\ell$, in the sense that $\alpha$ modulo $\ell$ generates $\mathbb{T}\left(\mathbb{F}_{\ell}\right)$.

In $\S 2$, we begin with the condition for given $\alpha \in \mathcal{O}_{K}$ to be primitive point modulo prime $\ell$ for the torus $\mathbb{T}_{K}$, where $K$ is an arbitrary imaginary quadratic field and $\ell$ is a rational prime which remains prime in $K$. The set $M_{\alpha}$ consisting of all primes $\ell$ having this property with respect to a fixed $\alpha$ is then characterized algebraically via a family of Galois extensions constructed from $\alpha$. In $\S 3$ we prove that $M_{\alpha}$ always has a density (assuming GRH) which can be given precisely. An application to elliptic curves is given in $\S 4$. Our method works well for elliptic curve $E$ over any finite field $\mathbb{F}_{r}$, and one can gather in this way information on the distributions of the degrees $\left[\mathbb{F}_{r}(E[\ell]): \mathbb{F}_{r}\right]$ as $\ell$ ranges over all prime numbers.

2. Primitive Points for Certain Two Dimensional Tori. Let $K$ be a fixed imaginary quadratic number field, with ring of integers $\mathcal{O}_{K} \subset K$. We use $\tau$ to denote the complex conjugation and in this section $\ell$ always stands for a rational prime number that stays prime in $K$. For $\alpha \in \mathcal{O}_{K} \backslash\{0\}, N(\alpha)=\alpha \alpha^{\tau}$ denotes its absolute norm, $\bar{\alpha}$ denotes the coset in $\left(\mathcal{O}_{K} / \ell \mathcal{O}_{K}\right)^{\star}$ containing $\alpha$ if $\operatorname{ord}_{\ell}(\alpha)=0$, and $o_{\ell}(\alpha)$ denotes the order of $\bar{\alpha}$ inside $\left(\mathcal{O}_{K} / \ell \mathcal{O}_{K}\right)^{\star}$. The set of all rational prime numbers is denoted by $\mathbb{P}$. Given $\alpha \in \mathcal{O}_{K} \backslash\{0\}$, we set $u=u(\alpha)=\alpha^{\tau} / \alpha$. The following straightforward Proposition is the starting point:

Proposition 2.1. Let $\ell$ be a rational prime that is inert(stays prime) in $K$ and $\operatorname{ord}_{\ell}(\alpha)=0$. Then $o_{\ell}(\alpha)=\ell^{2}-1$ if and only if $o_{\ell}(N(\alpha))=\ell-1$ and $o_{\ell}(u)=\ell+1$.

Proof. Note that as $\ell$ is inert in $K, \alpha^{\tau} \equiv \alpha^{\ell}(\bmod \ell)$. Thus $N(\alpha) \equiv \alpha^{\ell+1}$ and $u \equiv \alpha^{\ell-1}(\bmod \ell)$. Suppose that $o_{\ell}(\alpha)=\ell^{2}-1$. Then clearly we have $o_{\ell}(N(\alpha))=\ell-1$ and $o_{\ell}(u)=\ell+1$. Conversely, if $o_{\ell}(\alpha) \neq \ell^{2}-1$, then there exists a prime $q$ such that $q \mid \ell^{2}-1$ and $\alpha^{\frac{\ell^{2}-1}{q}} \equiv 1(\bmod \ell)$. If $q \mid \ell-1$, then $N(\alpha)^{\frac{\ell-1}{q}} \equiv \alpha^{\frac{\ell^{2}-1}{q}} \equiv 1(\bmod \ell)$; and if $q \mid \ell+1$, then $u^{\frac{\ell+1}{q}} \equiv \alpha^{\frac{\ell^{2}-1}{q}} \equiv 1(\bmod \ell)$. Therefore, $o_{\ell}(\alpha) \neq \ell^{2}-1$ implies either $o_{\ell}\left((N(\alpha)) \supsetneqq \ell-1\right.$ or $o_{\ell}(u) \supsetneqq \ell+1$.

Recall that $\mathbb{T}=\operatorname{Res}_{K / \mathbb{Q}} \mathbb{G}_{m / K}$ is the algebraic group over $\mathbb{Q}$ obtained from the multiplicative group $\mathbb{G}_{m}$ by restriction of scalars. We have $\alpha \in \mathcal{O}_{K} \backslash\{0\} \subset K^{\star}=\mathbb{T}(\mathbb{Q})$ and we are interested in the following set of primes:

$$
\begin{aligned}
M_{\alpha} & =\left\{\ell: \ell \text { rational prime that is inert in } K, \operatorname{ord}_{\ell}(\alpha)=0, o_{\ell}(\alpha)=\ell^{2}-1\right\} \\
& =\left\{\ell: \ell \text { rational prime that is inert in } K, \bar{\alpha} \text { generate } \mathbb{T}\left(\mathbb{F}_{\ell}\right)\right\} .
\end{aligned}
$$

Notations: Let $q, q^{\prime}$ be rational primes with $q^{\prime}$ odd. We introduce the following Galois number fields:

$E_{1}=\mathbb{Q}, F_{1}=K$.

$E_{q}=\mathbb{Q}\left(\boldsymbol{\mu}_{q}, \sqrt[q]{N(\alpha)}\right)$, where $\boldsymbol{\mu}_{q}$ is the group of $q$-th roots of unity.

$E_{m}=$ the compositum $\prod_{q \mid m} E_{q}$, for square free $m$.

$F_{q^{\prime}}=K\left(\boldsymbol{\mu}_{q^{\prime}}, \sqrt[q^{\prime}]{u}\right)$

$F_{n}=$ the compositum $\prod_{q^{\prime} \mid n} F_{q^{\prime}}$, for square free odd $n$.

$L_{m, n}=$ the compositum $E_{m} F_{n}$, for $m, n$ square free and $n$ is odd.

For Galois number fields $E / F,(\wp, E / F)$ will denote the Artin symbol whenever the prime $\wp$ in $F$ is unramified in $E$. We shall allow $\tau$ to stand also for the complex 
conjugation on $\mathbb{Q}\left(\boldsymbol{\mu}_{n}\right)$, i.e. $\tau(\xi)=\xi^{-1}$ for any $\xi \in \boldsymbol{\mu}_{n}$. Given square free $m, n$, with $n$ odd, we consider in particular the following subset of $\operatorname{Gal}\left(L_{m, n} / \mathbb{Q}\right)$ :

$$
C_{m, n}=\left\{\sigma \in \operatorname{Gal}\left(L_{m, n} / \mathbb{Q}\right):\left.\sigma\right|_{K}=\tau,\left.\sigma\right|_{E_{m}}=\mathrm{id},\left.\sigma\right|_{\mathbb{Q}\left(\boldsymbol{\mu}_{n}\right)}=\tau, \text { and } \sigma^{2}=\mathrm{id}\right\} .
$$

We have

Lemma 2.2. Let $\ell$ be a prime that is inert in $K / \mathbb{Q}$ and $q$ be a prime. Suppose $\operatorname{ord}_{\ell}(\alpha)=0$. Then the following conditions are equivalent:

(1) $q \mid(\ell-1)$ and $\bar{\alpha}^{\frac{\ell^{2}-1}{q}}=1$ in $\left(\mathcal{O}_{K} / \ell \mathcal{O}_{K}\right)^{\star}$.

(2) $\ell$ splits completely in $E_{q} / \mathbb{Q}$.

(3) $\ell$ is unramified in $L_{q, 1} / \mathbb{Q}$ and $\left(\ell, L_{q, 1} / \mathbb{Q}\right) \subseteq C_{q, 1}$.

Proof. It suffices to note that (1) amounts to $q \mid(\ell-1)$ and $N(\alpha)^{\frac{\ell-1}{q}} \equiv 1$ $(\bmod \ell)$. This is equivalent to $q \mid(\ell-1)$ and $x^{q} \equiv N(\alpha)(\bmod \ell)$ has a solution in $\mathbb{Z}$. Thus we have $(1) \Leftrightarrow(2)$. The rest follows from the definitions.

Lemma 2.3. Let $\ell$ be a prime that is inert in $K / \mathbb{Q}$ and $q^{\prime}$ be an odd prime. Suppose $\operatorname{ord}_{\ell}(\alpha)=0$. Then the following conditions are equivalent:

(1) $q^{\prime} \mid(\ell+1)$ and $\bar{\alpha}^{\frac{\ell^{2}-1}{q^{\prime}}}=1$ in $\left(\mathcal{O}_{K} / \ell \mathcal{O}_{K}\right)^{\star}$.

(2) $q^{\prime} \mid(\ell+1)$ and $\ell \mathcal{O}_{K}$ splits completely in $F_{q^{\prime}} / K$.

(3) $\ell$ is unramified in $L_{1, q^{\prime}} / \mathbb{Q}$ and $\left(\ell, L_{1, q^{\prime}} / \mathbb{Q}\right) \subseteq C_{1, q^{\prime}}$.

Proof. We first note that $(1) \Leftrightarrow q^{\prime} \mid \ell+1$ and $\bar{u}^{\frac{\ell+1}{q^{\prime}}}=1$ in $\left(\mathcal{O}_{K} / \ell \mathcal{O}_{K}\right)^{\star}$. This is equivalent to $q^{\prime} \mid(\ell+1)$ and $x^{q^{\prime}} \equiv \alpha(\bmod \ell)$ has a solution in $\mathcal{O}_{K}$, since $\left(\mathcal{O}_{K} / \ell \mathcal{O}_{K}\right)^{\star}$ is cyclic. Also it is equivalent to $q^{\prime} \mid(\ell+1)$ and $x^{q^{\prime}} \equiv \alpha^{\ell-1} \equiv u(\bmod \ell)$ has a solution in $\mathcal{O}_{K}$, because $q^{\prime} \nmid(\ell-1)$. Hence we obtain (1) $\Leftrightarrow(2)$. On the other hand $q^{\prime} \mid(\ell+1)$ if and only if $\left(\ell, \mathbb{Q}\left(\boldsymbol{\mu}_{q^{\prime}}\right) / \mathbb{Q}\right)=\tau$. If (2) holds, then $\ell$ is clearly unramified in $L_{1, q^{\prime}} / \mathbb{Q}$. Because $\ell \mathcal{O}_{K}$ splits completely in $L_{1, q^{\prime}} / K$, we have $\sigma^{2}=$ id, for all $\sigma \in\left(\ell, L_{1, q^{\prime}} / \mathbb{Q}\right)$. Thus $(2) \Rightarrow(3)$. Conversely, from $\sigma^{2}=$ id for all $\sigma \in\left(\ell, L_{1, q^{\prime}} / \mathbb{Q}\right)$, we obtain immediately that $\ell \mathcal{O}_{K}$ splits completely in $F_{q^{\prime}} / K$. Hence (3) $\Rightarrow(2)$.

Combining Lemmas 2.2, 2.3, we deduce the crucial:

COROLlary 2.4. Let $\ell$ be a rational prime which is inert in $K / \mathbb{Q}$ and $\operatorname{ord}_{\ell}(\alpha)=$ 0 . Then $\ell \in M_{\alpha}$ if and only if both the following two conditions hold: (1) For all prime $q$, if $\ell$ is unramified in $L_{q, 1}$, then $\left(\ell, L_{q, 1} / \mathbb{Q}\right) \nsubseteq C_{q, 1}$.

(2) For all odd prime $q^{\prime}$, if $\ell$ is unramified in $L_{1, q^{\prime}}$, then $\left(\ell, L_{1, q^{\prime}} / \mathbb{Q}\right) \nsubseteq C_{1, q^{\prime}}$.

From now on we make the further assumption that $\alpha$ is not a root of unity and $\operatorname{gcd}\left(\alpha, \alpha^{\tau}\right)=1$, i.e. $1 \in \alpha \mathcal{O}_{K}+\alpha^{\tau} \mathcal{O}_{K}$. The remaining part of this section is occupied by a detailed study of the Galois family $L_{m, n}$, together with the computation of $\# C_{m, n}$. All these are preliminaries needed for the main theorems of $\S 3$.

LEMMA 2.5. Let $m, n$ be square-free positive integers with $n$ odd. Let $s$ be the largest integer with the property that $N(\alpha) \in\left(\mathbb{Q}^{*}\right)^{s}$, and let $s^{\prime}$ be the largest integer with the property that $u \in\left(K^{\star}\right)^{s^{\prime}}$. Let $m_{1}=m / \operatorname{gcd}(m, s)$ and $n_{2}=n / \operatorname{gcd}\left(n, s^{\prime}\right)$. Suppose $\operatorname{gcd}(s, 6)=1$. Then

(a)

$$
\left[E_{m}: \mathbb{Q}\right]=\frac{m_{1} \phi(m)}{\left[k_{m} \cap \mathbb{Q}\left(\mu_{m}\right): \mathbb{Q}\right]},
$$

where $k_{m}=\mathbb{Q}($ resp. $\mathbb{Q}(\sqrt{N(\alpha)}))$ if $2 \nmid m($ resp. $2 \mid m)$. 
(b)

$$
\left[F_{n}: \mathbb{Q}\right]= \begin{cases}\frac{2 n_{2} \phi(n)}{3\left[K \cap \mathbb{Q}\left(\mu_{n}\right): \mathbb{Q}\right] .} & \text { if } K=\mathbb{Q}(\sqrt{-3}), 3 \mid n, \text { and } u \in\left(K\left(\boldsymbol{\mu}_{n}\right)^{\star}\right)^{3} \\ \frac{2 n_{2} \phi(n)}{\left[K \cap \mathbb{Q}\left(\mu_{n}\right): \mathbb{Q}\right]} & \text { otherwise. }\end{cases}
$$

Proof. Our argument is based on the following

Sublemma. Let $F$ be a field, $K_{1}$ a finite abelian extension of $F$, and $K_{2}$ be a finite extension of $F$ which is not Galois but with prime extension degree. Then $K_{1}, K_{2}$ are linearly disjoint over $F$ and $\left[K_{1} K_{2}: K_{1}\right]=\left[K_{2}: F\right]$.

(a) Suppose that $2 \nmid m$. For $q \mid m$, let $E_{m, q}=\mathbb{Q}\left(\boldsymbol{\mu}_{m}, \sqrt[q]{N(\alpha)}\right)$. Note that $\left[\mathbb{Q}(\sqrt[q]{N(\alpha)}: \mathbb{Q}]=1\right.$ or $q$ depending on whether $N(\alpha) \in\left(\mathbb{Q}^{\star}\right)^{q}$. By the Sublemma, we have therefore

$$
\left[E_{m, q}: \mathbb{Q}\left(\boldsymbol{\mu}_{m}\right)\right]= \begin{cases}1 & \text { if } N(\alpha) \in\left(\mathbb{Q}^{*}\right)^{q} \\ q & \text { otherwise. }\end{cases}
$$

Thus $E_{m, q}$ 's are linearly disjoint over $\mathbb{Q}\left(\boldsymbol{\mu}_{m}\right)$. Since $E_{m}$ is the compositum of $E_{m, q}$ 's, we have $\left[E_{m}: \mathbb{Q}\right]=\left[E_{m}: \mathbb{Q}\left(\boldsymbol{\mu}_{m}\right)\right]\left[\mathbb{Q}\left(\boldsymbol{\mu}_{m}\right): \mathbb{Q}\right]=m_{1} \phi(m)$.

Suppose that $2 \mid m$, write $m=2 m^{\prime}$. Then $m_{1}=m / \operatorname{gcd}(m, s)=2 \cdot m^{\prime} / \operatorname{gcd}\left(m^{\prime}, s\right)$ $=2 m_{1}^{\prime}$ and $E_{m}=E_{2} E_{m^{\prime}}$. For $q^{\prime} \mid m^{\prime}$, let $E_{m, q^{\prime}}=E_{2}\left(\boldsymbol{\mu}_{m^{\prime}}, \sqrt[q^{\prime}]{N(\alpha)}\right)$. Here one also has $\left[E_{2}\left(\sqrt[q^{\prime}]{N(\alpha)}\right): E_{2}\right]=1$ or $q^{\prime}$ depending on whether $N(\alpha) \in\left(\mathbb{Q}^{\star}\right)^{q^{\prime}}$. Consequently,

$$
\left[E_{m, q^{\prime}}: E_{2}\left(\boldsymbol{\mu}_{m^{\prime}}\right)\right]= \begin{cases}1 & \text { if } N(\alpha) \in\left(\mathbb{Q}^{\star}\right)^{q^{\prime}} \\ q & \text { otherwise. }\end{cases}
$$

The $E_{m, q^{\prime}}$ 's are linearly disjoint over $E_{2}\left(\boldsymbol{\mu}_{m^{\prime}}\right)$ and we have

$$
\begin{aligned}
{\left[E_{m}: \mathbb{Q}\right] } & =\left[E_{m}: E_{2}\left(\boldsymbol{\mu}_{m^{\prime}}\right)\right]\left[E_{2}\left(\boldsymbol{\mu}_{m^{\prime}}\right): \mathbb{Q}\right] \\
& =\frac{m_{1}}{2} \frac{\left[E_{2}: \mathbb{Q}\right]\left[\mathbb{Q}\left(\boldsymbol{\mu}_{m^{\prime}}\right): \mathbb{Q}\right]}{\left[E_{2} \cap \mathbb{Q}\left(\mu_{m^{\prime}}\right): \mathbb{Q}\right]} \\
& =\frac{m_{1} \phi(m)}{\left[E_{2} \cap \mathbb{Q}\left(\mu_{m}\right): \mathbb{Q}\right]} .
\end{aligned}
$$

(b) For $q^{\prime} \mid n$, let $F_{n, q^{\prime}}=K\left(\boldsymbol{\mu}_{n}, \sqrt[q]{u}\right)$. Note that if $q^{\prime} \nmid s^{\prime}$, then $K\left(\sqrt[q^{\prime}]{u}\right)$ is not Galois over $K$ except that $K=\mathbb{Q}(\sqrt{-3})$ and $q^{\prime}=3$. Also one has that $\left[K\left(\sqrt[q^{\prime}]{u)}\right.\right.$ : $K]=1$ or $q^{\prime}$ depending on whether $u \in\left(K^{\star}\right)^{q^{\prime}}$. By the Sublemma, we have $\left[F_{n, q^{\prime}}\right.$ : $\left.K\left(\boldsymbol{\mu}_{n}\right)\right]=q^{\prime} / \operatorname{gcd}\left(q^{\prime}, s^{\prime}\right)$ except when $K=\mathbb{Q}(\sqrt{-3})$ and $q^{\prime}=3$. If $K=\mathbb{Q}(\sqrt{-3})$ and $3 \mid n$, then

$$
\left[F_{n, 3}: K\left(\boldsymbol{\mu}_{n}\right)\right]= \begin{cases}1 & \text { if } u \in\left(K\left(\boldsymbol{\mu}_{n}\right)^{\star}\right)^{3} \\ 3 & \text { if } u \notin\left(K\left(\boldsymbol{\mu}_{n}\right)^{\star}\right)^{3}\end{cases}
$$

Thus the $F_{n, q^{\prime}}$ 's are linearly disjoint over $K\left(\boldsymbol{\mu}_{n}\right)$ and we have:

$$
\left[F_{n}: K\left(\boldsymbol{\mu}_{n}\right)\right]= \begin{cases}\frac{n_{2}}{3} & \text { if } K=\mathbb{Q}(\sqrt{-3}), 3 \mid n, \text { and } u \in\left(K\left(\boldsymbol{\mu}_{n}\right)^{\star}\right)^{3}, \\ n_{2} & \text { otherwise. }\end{cases}
$$

Therefore,

$$
\begin{aligned}
{\left[F_{n}: \mathbb{Q}\right] } & =\left[F_{n}: K\left(\boldsymbol{\mu}_{n}\right)\right]\left[K\left(\boldsymbol{\mu}_{n}\right): \mathbb{Q}\right] \\
& = \begin{cases}\frac{2 n_{2} \phi(n)}{3\left[K \cap \mathbb{Q}\left(\boldsymbol{\mu}_{n}\right): \mathbb{Q}\right]} & \text { if } K=\mathbb{Q}(\sqrt{-3}), 3 \mid n, \text { and } u \in\left(K\left(\boldsymbol{\mu}_{n}\right)^{\star}\right)^{3}, \\
\frac{2 n_{2} \phi(n)}{\left[K \cap \mathbb{Q}\left(\boldsymbol{\mu}_{n}\right): \mathbb{Q}\right]} & \text { otherwise. }\end{cases}
\end{aligned}
$$


LEMMA 2.6. Let $m, n$ be square-free positive integers with $n$ odd and $\operatorname{gcd}(m, n)=$ 1. Suppose further that $\alpha$ satisfies all the conditions in Lemma 2.5. If $K=\mathbb{Q}(\sqrt{-3})$, $3 \mid n$ and $u \in\left(K\left(\boldsymbol{\mu}_{m n}\right)^{\star}\right)^{3} \backslash\left(K\left(\boldsymbol{\mu}_{n}\right)^{\star}\right)^{3}$, then $E_{m} \cap F_{n}=k_{m}\left(\boldsymbol{\mu}_{m}\right) \cap K\left(\boldsymbol{\mu}_{n}, \sqrt[3]{u}\right)$ and

$$
\left[E_{m} \cap F_{n}: \mathbb{Q}\right]=\frac{3\left[K k_{m} \cap \mathbb{Q}\left(\boldsymbol{\mu}_{m n}\right): \mathbb{Q}\right]}{\left[k_{m} \cap \mathbb{Q}\left(\boldsymbol{\mu}_{m}\right): \mathbb{Q}\right]\left[K \cap \mathbb{Q}\left(\boldsymbol{\mu}_{n}\right): \mathbb{Q}\right]} .
$$

Otherwise, $E_{m} \cap F_{n}=k_{m}\left(\boldsymbol{\mu}_{m}\right) \cap K\left(\boldsymbol{\mu}_{n}\right)$ and

$$
\left[E_{m} \cap F_{n}: \mathbb{Q}\right]=\frac{\left[K k_{m} \cap \mathbb{Q}\left(\boldsymbol{\mu}_{m n}\right): \mathbb{Q}\right]}{\left[k_{m} \cap \mathbb{Q}\left(\boldsymbol{\mu}_{m}\right): \mathbb{Q}\right]\left[K \cap \mathbb{Q}\left(\boldsymbol{\mu}_{n}\right): \mathbb{Q}\right]}
$$

(Recall that $k_{m}=\mathbb{Q}($ resp. $\mathbb{Q}(\sqrt{N(\alpha)}))$ if $2 \nmid m($ resp. $2 \mid m)$.)

Proof. First we contend that $E_{m} \cap K k_{m}\left(\boldsymbol{\mu}_{m n}\right)=k_{m}\left(\boldsymbol{\mu}_{m}\right)$. Also that $F_{n} \cap$ $K k_{m}\left(\boldsymbol{\mu}_{m n}\right)=K\left(\boldsymbol{\mu}_{n}\right)$ except when $K=\mathbb{Q}(\sqrt{-3}), 3 \mid n, u \in\left(K\left(\boldsymbol{\mu}_{m n}\right)^{\star}\right)^{3}$ but $u \notin$ $\left(K\left(\boldsymbol{\mu}_{n}\right)^{\star}\right)^{3}$.

Since $\operatorname{gcd}(m, n)=1, K k_{m}\left(\boldsymbol{\mu}_{m n}, \sqrt[m]{N(\alpha)}\right)$ and $K k_{m}\left(\boldsymbol{\mu}_{m n}, \sqrt[n]{u}\right)$ are linearly disjoint over $K k_{m}\left(\boldsymbol{\mu}_{m n}\right)$. Observe that $E_{m} \subset K k_{m}\left(\boldsymbol{\mu}_{m n}, \sqrt[m]{N(\alpha)}\right)$ and $F_{n} \subset$ $K k_{m}\left(\boldsymbol{\mu}_{m n}, \sqrt[n]{u}\right)$. Hence

$$
E_{m} \cap F_{n} \subseteq K k_{m}\left(\boldsymbol{\mu}_{m n}, \sqrt[m]{N(\alpha)}\right) \cap K k_{m}\left(\boldsymbol{\mu}_{m n}, \sqrt[n]{u}\right)=K k_{m}\left(\boldsymbol{\mu}_{m n}\right)
$$

Note that for odd prime $q, N(\alpha) \in\left(\mathbb{Q}^{\star}\right)^{q}$ if and only if $N(\alpha) \in\left(\left(K k_{m}\right)^{\star}\right)^{q}$. Similar to the proof of Lemma 2.5(a), one has

$$
\left[K k_{m}\left(\boldsymbol{\mu}_{m n}, \sqrt[m]{N(\alpha)}\right): K k_{m}\left(\boldsymbol{\mu}_{m n}\right)\right]=\frac{m_{1}}{\operatorname{gcd}(2, m)}=\left[E_{m}: k_{m}\left(\boldsymbol{\mu}_{m}\right)\right]
$$

and therefore $E_{m} \cap K k_{m}\left(\boldsymbol{\mu}_{m n}\right)=k_{m}\left(\boldsymbol{\mu}_{m}\right)$.

Similarly, because $N(\alpha)$ is divisible only by splitting primes, if $q^{\prime} \nmid s^{\prime}$, then $K k_{m}\left(\sqrt[q^{\prime}]{ }\right)$ is not Galois over $K k_{m}$ except when $K=\mathbb{Q}(\sqrt{-3})$ and $q^{\prime}=3$. Thus we have

$$
\left[K k_{m}\left(\boldsymbol{\mu}_{m n}, \sqrt[n]{u}\right): K k_{m}\left(\boldsymbol{\mu}_{m n}\right)\right]=\left\{\begin{array}{cl}
\frac{n_{2}}{3} & \text { if } K=\mathbb{Q}(\sqrt{-3}), 3 \mid n, u \in\left(K\left(\boldsymbol{\mu}_{m n}\right)^{\star}\right)^{3} \\
n_{2} & \text { otherwise. }
\end{array}\right.
$$

Therefore, $F_{n} \cap K k_{m}\left(\boldsymbol{\mu}_{m n}\right)=K\left(\boldsymbol{\mu}_{n}\right)$ unless $K=\mathbb{Q}(\sqrt{-3}), 3 \mid n$, and $u \in\left(K\left(\boldsymbol{\mu}_{m n}\right)^{\star}\right)^{3} \backslash$ $\left(K\left(\boldsymbol{\mu}_{n}\right)^{\star}\right)^{3}$. In the last mentioned case, it is easy to check that $\left[F_{n} \cap K k_{m}\left(\boldsymbol{\mu}_{m n}\right)\right.$ : $\left.K\left(\boldsymbol{\mu}_{n}\right)\right]=3$ and thus $F_{n} \cap K k_{m}\left(\boldsymbol{\mu}_{m n}\right)=K\left(\boldsymbol{\mu}_{n}, \sqrt[3]{u}\right)$.

If we are in the case $K=\mathbb{Q}(\sqrt{-3}), 3 \mid n, u \in\left(K\left(\boldsymbol{\mu}_{m n}\right)^{\star}\right)^{3} \backslash\left(K\left(\boldsymbol{\mu}_{n}\right)^{\star}\right)^{3}$, what we obtain is

$$
\begin{aligned}
& \left.\left[E_{m} \cap F_{n}: \mathbb{Q}\right]=\left[\left(E_{m} \cap K k_{m}\left(\boldsymbol{\mu}_{m n}\right)\right) \cap\left(F_{n} \cap K k_{m}\left(\boldsymbol{\mu}_{m n}\right)\right): \mathbb{Q}\right)\right] \\
& =\left[k_{m}\left(\boldsymbol{\mu}_{m}\right) \cap K\left(\boldsymbol{\mu}_{n}, \sqrt[3]{u}\right): \mathbb{Q}\right]=\frac{\left[k_{m}\left(\boldsymbol{\mu}_{m}\right): \mathbb{Q}\right]\left[K\left(\boldsymbol{\mu}_{n}, \sqrt[3]{u}\right): \mathbb{Q}\right]}{\left[K k_{m}\left(\boldsymbol{\mu}_{m n}, \sqrt[3]{u}\right): \mathbb{Q}\right]} \\
& =\frac{3\left[k_{m}\left(\boldsymbol{\mu}_{m}\right): \mathbb{Q}\right]\left[K\left(\boldsymbol{\mu}_{n}\right): \mathbb{Q}\right]}{\left[K k_{m}\left(\boldsymbol{\mu}_{m n}\right): \mathbb{Q}\right]} \\
& =3 \cdot \frac{\left[k_{m}: \mathbb{Q}\right]\left[\mathbb{Q}\left(\boldsymbol{\mu}_{m}\right): \mathbb{Q}\right]}{\left[k_{m} \cap \mathbb{Q}\left(\boldsymbol{\mu}_{m}\right): \mathbb{Q}\right]} \frac{[K: \mathbb{Q}]\left[\mathbb{Q}\left(\boldsymbol{\mu}_{n}\right): \mathbb{Q}\right]}{\left[K \cap \mathbb{Q}\left(\boldsymbol{\mu}_{n}\right): \mathbb{Q}\right]} \frac{\left[K k_{m} \cap \mathbb{Q}\left(\boldsymbol{\mu}_{m n}\right): \mathbb{Q}\right]}{\left[K k_{m}: \mathbb{Q}\right]\left[\mathbb{Q}\left(\boldsymbol{\mu}_{m n}\right): \mathbb{Q}\right]} \\
& =\frac{3\left[K k_{m} \cap \mathbb{Q}\left(\boldsymbol{\mu}_{m n}\right): \mathbb{Q}\right]}{\left[k_{m} \cap \mathbb{Q}\left(\boldsymbol{\mu}_{m}\right): \mathbb{Q}\right]\left[K \cap \mathbb{Q}\left(\boldsymbol{\mu}_{n}\right): \mathbb{Q}\right]} .
\end{aligned}
$$


On the other hand, in all other cases, we have

$$
\begin{aligned}
& {\left[E_{m} \cap F_{n}: \mathbb{Q}\right]=\left[k_{m}\left(\boldsymbol{\mu}_{m}\right) \cap K\left(\boldsymbol{\mu}_{n}\right): \mathbb{Q}\right]=\frac{\left[k_{m}\left(\boldsymbol{\mu}_{m}\right): \mathbb{Q}\right]\left[K\left(\boldsymbol{\mu}_{n}\right): \mathbb{Q}\right]}{\left[K k_{m}\left(\boldsymbol{\mu}_{m n}\right): \mathbb{Q}\right]}} \\
& =\frac{\left[k_{m}: \mathbb{Q}\right]\left[\mathbb{Q}\left(\boldsymbol{\mu}_{m}\right): \mathbb{Q}\right]}{\left[k_{m} \cap \mathbb{Q}\left(\boldsymbol{\mu}_{m}\right): \mathbb{Q}\right]} \frac{[K: \mathbb{Q}]\left[\mathbb{Q}\left(\boldsymbol{\mu}_{n}\right): \mathbb{Q}\right]}{\left[K \cap \mathbb{Q}\left(\boldsymbol{\mu}_{n}\right): \mathbb{Q}\right]} \frac{\left[K k_{m} \cap \mathbb{Q}\left(\boldsymbol{\mu}_{m n}\right): \mathbb{Q}\right]}{\left[K k_{m}: \mathbb{Q}\right]\left[\mathbb{Q}\left(\boldsymbol{\mu}_{m n}\right): \mathbb{Q}\right]} \\
& =\frac{\left[K k_{m} \cap \mathbb{Q}\left(\boldsymbol{\mu}_{m n}\right): \mathbb{Q}\right]}{\left[k_{m} \cap \mathbb{Q}\left(\boldsymbol{\mu}_{m}\right): \mathbb{Q}\right]\left[K \cap \mathbb{Q}\left(\boldsymbol{\mu}_{n}\right): \mathbb{Q}\right]} \cdot \quad
\end{aligned}
$$

LEMMA 2.7. Let $m, n$ be square-free positive integers with $n$ odd. Suppose further that $\alpha$ satisfies all the conditions in Lemma 2.5. Let $c_{m, n}=\# C_{m, n}$. Then we have

$$
c_{m, n}= \begin{cases}1 & \text { if } E_{m} \cap F_{n} \text { is totally real, } \\ 0 & \text { otherwise. }\end{cases}
$$

In particular, if $\operatorname{gcd}(m, n) \neq 1$, then $c_{m, n}=0$.

Proof. Suppose that $E_{m} \cap F_{n}$ is not totally real. Then it is clear that $C_{m, n}=\emptyset$ and thus $c_{m, n}=0$.

Now suppose that $E_{m} \cap F_{n}$ is totally real. Then $\operatorname{gcd}(m, n)=1$. Note that $C_{m, n} \subseteq \operatorname{Gal}\left(L_{m, n} / E_{m}\right) \subseteq \operatorname{Gal}\left(L_{m, n} / E_{m} \cap F_{n}\right) \subseteq \operatorname{Gal}\left(L_{m, n} / \mathbb{Q}\right)$. Recall that $L_{m, n}$ is the compositum of $E_{m}, F_{n}$ and thus one has the following isomorphism

$$
\begin{aligned}
\operatorname{Gal}\left(L_{m, n} / E_{m} \cap F_{n}\right) & \stackrel{\sim}{\longrightarrow} \operatorname{Gal}\left(E_{m} / E_{m} \cap F_{n}\right) \times \operatorname{Gal}\left(F_{n} / E_{m} \cap F_{n}\right) \\
\sigma & \mapsto\left(\sigma_{1}, \sigma_{2}\right)=\left(\left.\sigma\right|_{E_{m}},\left.\sigma\right|_{F_{n}}\right) .
\end{aligned}
$$

Embedding $F_{n}$ into $\mathbb{C}$, and restricting the complex conjugation $\tau$ to $F_{n}$, since $E_{m} \cap F_{n}$ is totally real, we may extend $\left.\tau\right|_{F_{n}}$ to an element in $C_{m, n}$. It suffices to show that $\sigma \in C_{m n}$ if and only if $\sigma_{2}=\left.\tau\right|_{F_{n}}$. Suppose $\sigma \in C_{m, n}$. Then $\sigma_{1}=$ id. Let $\zeta_{n}$ be a fixed primitive $n$-th root of unity. Suppose $\sigma_{2}(\sqrt[n]{u})=\zeta_{n}^{i} \frac{1}{\sqrt[n]{u}}$ for some fixed $i, 0 \leq i \leq n-1$. Then $\sqrt[n]{u}=\sigma_{2}^{2}(\sqrt[n]{u})=\zeta_{n}^{-2 i} \sqrt[n]{u}$. As $n$ is odd, it follows that $i=0$ and $\sigma_{2}$ is unique on $F_{n}$.

Let $d_{m, n}$ denote the extension degree of $L_{m, n}$ over $\mathbb{Q}$. Then we have

LEMMA 2.8. Let $m, n$ be square-free positive integers with $n$ odd and $\operatorname{gcd}(m, n)=$ 1. Suppose further that $\alpha$ satisfies all the conditions in Lemma 2.5. Then

$$
d_{m, n}= \begin{cases}\frac{2 m_{1} n_{2} \phi(m n)}{3\left[K k_{m} \cap \mathbb{Q}\left(\boldsymbol{\mu}_{m n}\right): \mathbb{Q}\right]} & \text { if } K=\mathbb{Q}(\sqrt{-3}), 3 \mid n, \text { and } u \in\left(K\left(\boldsymbol{\mu}_{m n}\right)^{\star}\right)^{3}, \\ \frac{2 m_{1} n_{2} \phi(m n)}{\left[K k_{m} \cap \mathbb{Q}\left(\mu_{m n}\right): \mathbb{Q}\right]} & \text { otherwise. }\end{cases}
$$

Proof. Recall the fact that $L_{m, n}$ is the compositum of $E_{m}$ and $F_{n}$. Combining Lemma 2.5 and Lemma 2.6, we have, if $K=\mathbb{Q}(\sqrt{-3}), 3 \mid n$, and $u \in\left(K\left(\boldsymbol{\mu}_{m n}\right)^{\star}\right)^{3}$, then

$$
\begin{aligned}
d_{m, n} & =\left[L_{m, n}: \mathbb{Q}\right]=\left[E_{m}: \mathbb{Q}\right]\left[F_{n}: \mathbb{Q}\right] /\left[E_{m} \cap F_{n}: \mathbb{Q}\right] \\
& =\frac{2 m_{1} n_{2} \phi(m n)}{3\left[K k_{m} \cap \mathbb{Q}\left(\boldsymbol{\mu}_{m n}\right): \mathbb{Q}\right]} .
\end{aligned}
$$

Otherwise, $d_{m, n}=\frac{2 m_{1} n_{2} \phi(m n)}{\left[K k_{m} \cap \mathbb{Q}\left(\boldsymbol{\mu}_{m n}\right): \mathbb{Q}\right]}$.

Let $D_{K}$ denote the absolute value of the discriminant of the imaginary quadratic field $K$, and let $D_{m, n}$ denote the absolute value of the absolute discriminant of the number field $L_{m, n}$. Then we have 
LEMma 2.9. $D_{m, n} \mid D_{K}^{m_{1} n_{2} \phi(m) \phi(n)}(N(\alpha) m n)^{4 m_{1} n_{2} \phi(m) \phi(n)}$.

Proof. It is routine to compute that the relative discriminant of $E_{q} / \mathbb{Q}$ and

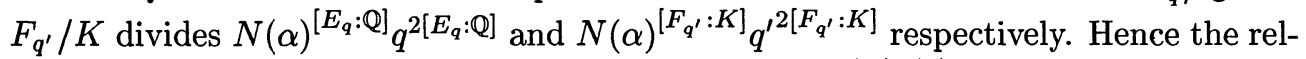
ative discriminant of $L_{m n} / K$ divides $(N(\alpha) m n)^{2 m_{1} n_{2} \phi(m) \phi(n)}$. Consequently $D_{m, n}$ | $D_{K}^{m_{1} n_{2} \phi(m) \phi(n)}(N(\alpha) m n)^{4 m_{1} n_{2} \phi(m) \phi(n)}$.

3. Existence and positivity of the density. Given a set $M \subset \mathbb{P}$, we are interested in the following limit:

$$
\lim _{x \rightarrow \infty} \frac{\#\{\ell \in M: \ell \leq x\}}{x / \log x} .
$$

If this limit exists, its value is called the density of $M$, and will be denoted by $\operatorname{den}(M)$. We are going to prove that if $\alpha \in K$ satisfies certain conditions, then the set $M_{\alpha}$ introduced in $\S \mathbf{2}$, has a positive density. The structure of our proof follows that of Hooley[2], c.f. also Murty[4].

For pimes $q, q^{\prime}$ with $q^{\prime}$ odd, we define two sets

$S_{q}=\left\{\ell \in \mathbb{P}: \ell\right.$ is inert in $K, \ell \mathcal{O}_{K}$ is unramified in $L_{q, 1}$, and $\left.\left(\ell, L_{q, 1}\right) \in C_{q, 1} \cdot\right\}$,

$T_{q^{\prime}}=\left\{\ell \in \mathbb{P}: \ell\right.$ is inert in $K, \ell \mathcal{O}_{K}$ is unramified in $L_{1, q^{\prime}}$, and $\left.\left(\ell, L_{1, q^{\prime}}\right) \in C_{1, q^{\prime}}.\right\}$.

Note that given a rational prime $\ell$, there are only finitely many $q$ 's such that $\ell \in S_{q}$ and also there are only finitely many $q^{\prime}$ 's such that $\ell \in T_{q^{\prime}}$. We define $R(\ell)$ to be the compositum

$$
R(\ell)=\prod_{q} L_{q, 1} \cdot \prod_{q^{\prime}} L_{1, q^{\prime}}
$$

where $q$ runs through primes satisfying $\ell \in S_{q}$ and $q^{\prime}$ runs through odd primes satisfying $\ell \in T_{q^{\prime}}$.

We are interested in the lattice of the fields $L_{m, n}{ }^{\prime} s$ where $m, n$ are square-free positive integers with $n$ odd, partially ordered by

$$
L_{m, n} \preceq L_{m^{\prime}, n^{\prime}} \text { if and only if } m^{\prime} \mid m \text { and } n^{\prime} \mid n \text {. }
$$

This lattice will be denoted by $\mathcal{L}$. Given $L \in \mathcal{L}$, we also introduce the functions:

$$
\begin{aligned}
f(x, L) & =\#\{\ell: \ell \in \mathbb{P}, \ell \leq x, \ell \text { is inert in } K / \mathbb{Q}, \text { and } R(\ell)=L\}, \\
\pi_{1}(x, L) & =\#\{\ell: \ell \in \mathbb{P}, \ell \leq x, \ell \text { is inert in } K / \mathbb{Q}, \text { and } R(\ell) \supseteq L\} .
\end{aligned}
$$

For $L \in \mathcal{L}$, it is clear that $\pi_{1}(x, L)=\sum_{L^{\prime} \preceq L} f\left(x, L^{\prime}\right)$. From Möbius inversion, we have $f(x, L)=\sum_{L^{\prime} \preceq L} \mu\left(L^{\prime}, L\right) \pi_{1}\left(x, L^{\prime}\right)$ where $\mu\left(L^{\prime}, L\right)$ is the Möbius function of the lattice L. (c.f. [5], Proposition 2.) In particular,

$$
f(x, K)=\sum \mu\left(L_{m, n}, K\right) \pi_{1}\left(x, L_{m, n}\right)=\sum \mu(m) \mu(n) \pi_{1}\left(x, L_{m, n}\right)
$$

where $m, n$ run through square-free positive integers with $n$ odd.

We will use the following effective version of Chebotarev Density Theorem.

Theorem 3.1. Let $L / \mathbb{Q}$ be finite Galois extension with Galois group $G$, and $C$ a union of conjugacy classes of $G$. Let $\pi_{C}(x, L / \mathbb{Q})=\#\{p: p$ is a prime unramified in $L / \mathbb{Q}, p \leq x$, and $(p, L / \mathbb{Q}) \subseteq C\}$. Assume GRH holds for the Dedekind zeta function 
of $L$. Then there exists a positive constant $A_{0}$ (independent of $C, L$ ) such that for every $x>2$,

$$
\left|\pi_{C}(x, L / \mathbb{Q})-\frac{\# C}{\# G} L i(x)\right| \leq A_{0}\left(\frac{\# C}{\# G} \sqrt{x} \log \left(D_{L} x^{[L: \mathbb{Q}]}\right)\right)
$$

where $L i(x)$ is the logarithmic integral $L i(x)=\int_{2}^{x} \frac{d t}{\log t} \sim \frac{x}{\log x}$ as $x \rightarrow \infty$.

(c.f. [6], Theorem 4.)

The existence of density for $M_{\alpha}$ is contained in the following

THEOREM 3.2. Given $\alpha \in \mathcal{O}_{K} \backslash \mathcal{O}_{K}{ }^{\star}$ with $\operatorname{gcd}\left(\alpha, \alpha^{\tau}\right)=1$. Let $s$ be the largest integer such that $N(\alpha) \in\left(\mathbb{Q}^{\star}\right)^{s}$. Assume that $\operatorname{gcd}(s, 6)=1$ and furthermore GRH holds. Then $\operatorname{den}\left(M_{\alpha}\right)$ exists and is given by

$$
\operatorname{den}\left(M_{\alpha}\right)=\sum_{m, n} \frac{\mu(m) \mu(n) c_{m, n}}{d_{m, n}}
$$

where in the sum $m, n$ runs through all square free positive integers, $n$ is required to be odd.

Remark. The condition $\operatorname{gcd}(s, 6)=1$ in Theorem 3.2 is not essential. If $2 \mid s$ or $3 \mid s$, one can still prove such an identiy with both sides equal 0 .

Proof of Theorem 3.2. First note that $\#\left\{\ell \in M_{\alpha}: \ell \leq x\right\}=f(x, K)$. Define $N(x, y)=\#\left\{\ell: \ell \in \mathbb{P}, \ell \leq x, \ell\right.$ is inert in $K, \ell \notin S_{q}$ and $\ell \notin T_{q^{\prime}}$ for all $\left.q, q^{\prime} \leq y.\right\}$. Recall that for any $\sigma \in \operatorname{Gal}\left(L_{m, n} / \mathbb{Q}\right)$,

$$
\left.\sigma \in C_{m, n} \Longleftrightarrow \sigma\right|_{L_{q, 1}} \in C_{q, 1} \text { for all } q \mid m \text { and }\left.\sigma\right|_{L_{1, q^{\prime}}} \in C_{1, q^{\prime}} \text { for all } q^{\prime} \mid n \text {. }
$$

Then it is clear that $f(x, K) \leq N(x, y)$ and

$$
N(x, y)=\sum_{m, n}^{\prime} \mu(m) \mu(n) \pi_{1}\left(x, L_{m, n}\right)
$$

where the dash on the sum indicates that all the prime divisors of $m n$ are $\leq y$. Note that $\pi_{1}\left(x, L_{m, n}\right)=\pi_{C_{m, n}}\left(x, L_{m, n}\right)$. Then applying Theorem 3.1 we can find a positive absolute constant $A_{0}$ such that for all $x>2$,

$$
\left|\pi_{1}\left(x, L_{m, n}\right)-\frac{c_{m, n}}{d_{m, n}} \operatorname{Li}(x)\right| \leq A_{0}\left(\frac{c_{m, n}}{d_{m, n}} \sqrt{x} \log \left(D_{m, n} x^{d_{m, n}}\right)\right) .
$$

Now define $M\left(x, y_{1}, y_{2}\right)=\#\left\{\ell: \ell \in \mathbb{P}, \ell \leq x, \ell \in S_{q}\right.$ for some $q \in\left[y_{1}, y_{2}\right]$ or $\ell \in T_{q^{\prime}}$ for some $\left.q^{\prime} \in\left[y_{1}, y_{2}\right].\right\}$. Then

$$
\text { (1) } f(x, K) \geq N(x, y)-M(x, y, x+1) \text {. }
$$

Claim 1: $M\left(x, \frac{\sqrt{x}}{\log ^{2} x}, \sqrt{x} \log x\right)=o\left(\frac{x}{\log x}\right)$.

Proof. The left-hand side is bounded by

$$
\sum_{\sqrt{x} / \log ^{2}} \pi_{x<q<\sqrt{x} \log x}\left(x, L_{q, 1}\right)+\sum_{\sqrt{x} / \log ^{2} x<q^{\prime}<\sqrt{x} \log x} \pi_{1}\left(x, L_{1, q^{\prime}}\right) .
$$

If $\ell$ contributes a count of 1 to $\pi_{1}\left(x, L_{q, 1}\right)$ then $\ell \equiv 1(\bmod q)$; if $\ell$ contributes a count of 1 to $\pi_{1}\left(x, L_{1, q^{\prime}}\right)$ then $\ell \equiv-1\left(\bmod q^{\prime}\right)$. Hence $\pi_{1}\left(x, L_{q, 1}\right)$ is bounded by $\#\{\ell: \ell \leq x, \ell \equiv 1(\bmod q)\}$ and $\pi_{1}\left(x, L_{1, q^{\prime}}\right)$ is bounded by $\#\{\ell: \ell \leq x, \ell \equiv-1$ $\left.\left(\bmod q^{\prime}\right)\right\}$. 
By the Brun-Titchmarsh theorem, for any $b \in \mathbb{Z}$, there is an absolute constant $B$ such that for $q<x$,

$$
\#\{\ell: \ell \leq x, \ell \equiv b \quad \bmod q\} \leq B \frac{x}{(q-1) \log (x / q)} .
$$

Therefore we have

$$
\begin{aligned}
M\left(x, \frac{\sqrt{x}}{\log ^{2} x}, \sqrt{x} \log x\right) & \leq 2 \cdot \sum_{\sqrt{x} / \log ^{2} x<q<\sqrt{x} \log x} B \frac{x}{(q-1) \log (x / q)} \\
& \leq \frac{x}{\log x} \cdot O\left(\sum \frac{1}{q}\right) \\
& \leq \frac{x}{\log ^{2} x} \cdot O\left(\sum \frac{\log q}{q}\right) \\
& \leq O\left(\frac{x \log \log x}{\log ^{2} x}\right)=o\left(\frac{x}{\log x}\right) .
\end{aligned}
$$

Claim 2: $M\left(x, \frac{\sqrt{x}}{\log ^{2} x}, x+1\right)=o\left(\frac{x}{\log x}\right)$.

Proof. Note that one can write

$$
M\left(x, \frac{\sqrt{x}}{\log ^{2} x}, x+1\right)=M\left(x, \frac{\sqrt{x}}{\log ^{2} x}, \sqrt{x} \log x\right)+M(x, \sqrt{x} \log x, x+1) .
$$

By Claim 1, it suffices to show that $M(x, \sqrt{x} \log x, x+1)=o\left(\frac{x}{\log x}\right)$. Recall that if $\ell \in S_{q}$, then $\ell \equiv 1(\bmod q)$ and $N(\alpha)^{\frac{\ell-1}{q}} \equiv 1(\bmod \ell)$ in $\mathcal{O}_{K}$, which implies $\ell$ divides $N(\alpha)^{\frac{\ell-1}{q}}-1$; similarly if $\ell \in T_{q^{\prime}}$, then $\ell \equiv-1\left(\bmod q^{\prime}\right)$ and $u^{\frac{\ell+1}{q^{\prime}}} \equiv 1$ $(\bmod \ell)$ in $\mathcal{O}_{K}$, which implies $\ell \mid\left(\alpha^{\frac{\ell+1}{q^{\prime}}}-\alpha^{\tau \frac{\ell+1}{q^{\prime}}}\right)$. Since $\ell \leq x$ and $q, q^{\prime}>\sqrt{x} \log x$, $(\ell-1) / q,(\ell+1) / q^{\prime}<2 \sqrt{x} / \log x$. Let

$$
R_{1}=\prod_{k<2 \sqrt{x} / \log x}\left(N(\alpha)^{k}-1\right) \quad \text { and } \quad R_{2}=\prod_{k<2 \sqrt{x} / \log x}\left(\alpha^{k}-\left(\alpha^{\tau}\right)^{k}\right)^{2} .
$$

Note that $R_{1} \neq 0$ and $R_{2} \neq 0$. Then it is easy to see that $\ell \in S_{q}$ implies $\ell \mid R_{1}$ and also that $\ell \in T_{q^{\prime}}$ implies $\ell \mid R_{2}$. So $M(x, \sqrt{x} \log x, x+1)$ is bounded by the number of prime factors of $R_{1} R_{2}$, which is trivially $O\left(\log R_{1}+\log R_{2}\right)$. Observe that

$$
\begin{aligned}
\log R_{1} & \leq \sum_{k<2 \sqrt{x} / \log x} k \log N(\alpha)=O\left(\frac{x}{\log ^{2} x}\right), \text { and } \\
\log R_{2} & \leq \sum_{k<2 \sqrt{x} / \log x} k \log \sqrt{N(\alpha)}=O\left(\frac{x}{\log ^{2} x}\right) .
\end{aligned}
$$

Therefore $M(x, \sqrt{x} \log x, x+1)=o\left(\frac{x}{\log x}\right)$.

Claim 3: $M(x, y, x+1)$ is $o\left(\frac{x}{\log x}\right)$ provided $y=y(x) \rightarrow \infty$ as $x \rightarrow \infty$.

Proof. It suffices to show that $M\left(x, y, \frac{\sqrt{x}}{\log ^{2} x}\right)=o\left(\frac{x}{\log x}\right)$. We have:

$$
M\left(x, y, \frac{\sqrt{x}}{\log ^{2} x}\right) \leq \sum_{y<q<\frac{\sqrt{x}}{\log ^{2} x}} \pi_{1}\left(x, L_{q, 1}\right)+\sum_{y<q^{\prime}<\frac{\sqrt{x}}{\log ^{2} x}} \pi_{1}\left(x, L_{1, q^{\prime}}\right),
$$




$$
\begin{aligned}
& \sum_{y<q<\frac{\sqrt{x}}{\log ^{2} x}} \pi_{1}\left(x, L_{q, 1}\right) \leq \sum_{y<q<\frac{\sqrt{x}}{\log ^{2} x}} \frac{c_{q, 1}}{d_{q, 1}} \operatorname{Li}(x)+O\left(\frac{c_{q, 1}}{d_{q, 1}} \sqrt{x} \log D_{q, 1} x^{d_{q, 1}}\right) \\
\leq & \operatorname{Li}(x) \sum_{y<q<\frac{\sqrt{x}}{\log ^{2} x}} \frac{1}{q^{2}}+\sqrt{x} \cdot O\left(\sum_{y<q<\frac{\sqrt{x}}{\log ^{2} x}} \log q\right)+\sqrt{x} \log x \cdot O\left(\sum_{y<q<\frac{\sqrt{x}}{\log ^{2} x}} 1\right) .
\end{aligned}
$$

One also gets the same bound for the sum involving $\pi_{1}\left(x, L_{1, q^{\prime}}\right)$. Hence

$$
\begin{aligned}
M\left(x, y, \frac{\sqrt{x}}{\log ^{2} x}\right) & \leq 2 \cdot \operatorname{Li}(x) \sum_{y<q<\frac{\sqrt{x}}{\log ^{2} x}} \frac{1}{q^{2}}+2 \cdot \sqrt{x} \log x \cdot O\left(\sum_{y<q<\frac{\sqrt{x}}{\log ^{2} x}} 1\right) \\
& =o\left(\frac{x}{\log x}\right)+O\left(\sqrt{x} \log x\left(\frac{\sqrt{x}}{\log ^{2} x} / \log \frac{\sqrt{x}}{\log ^{2} x}\right)\right)=o\left(\frac{x}{\log x}\right) .
\end{aligned}
$$

Claim 4: If $y(x)=O(\log x)$, then

$$
N(x, y)=\sum_{m, n}^{\prime} \frac{\mu(m) \mu(n) c_{m, n}}{d_{m, n}} \frac{x}{\log x}+o\left(\frac{x}{\log x}\right) .
$$

Proof. Applying the effective Cebotarev Density Theorem to those fields $L_{m, n}$, and union of conjugacy classes $C_{m, n}$, with all prime divisors of $m n$ bounded by $y$. Summing together the error terms, we have:

$$
\begin{aligned}
& O\left(\sum_{m, n}^{\prime} \frac{c_{m, n}}{d_{m, n}} \sqrt{x} \log D_{m, n} x^{d_{m, n}}\right)=O\left(\sqrt{x} \sum_{m, n}^{\prime} \log m n\right)+O\left(\sqrt{x} \log x \sum_{m, n}^{\prime} 1\right) \\
& =O\left(2^{2 t} \sqrt{x} \log y+2^{2 t} \sqrt{x} \log x\right) \\
& =O\left(2^{2 t} \sqrt{x} \log x\right)=o\left(\frac{x}{\log x}\right),
\end{aligned}
$$

where $t$ is the number of rational primes $\leq y$, thus $t=O\left(\frac{y}{\log y}\right)$.

Using Lemmas 2.7 and 2.8 we see that the series $\sum_{m, n} \frac{\mu(m) \mu(n) c_{m, n}}{d_{m, n}}$ is absolutely convergent. Now choose $y$ properly $(y=O(\log x))$, combining (1), Claim 3 and Claim 4 , we arrive at

$$
f(x, K)=\sum_{m, n} \frac{\mu(m) \mu(n) c_{m, n}}{d_{m, n}} \frac{x}{\log x}+o\left(\frac{x}{\log x}\right) .
$$

Therefore we conclude that $\operatorname{den}\left(M_{\alpha}\right)=\sum_{m, n} \frac{\mu(m) \mu(n) c_{m, n}}{d_{m, n}}$.

We are particularly interested in the case $N(\alpha)=p^{s}$, where $p$ is a prime splitting in the imaginary quadratic field $K$. As in $\S \mathbf{2}$, the case $K=\mathbb{Q}(\sqrt{-3})=K\left(\boldsymbol{\mu}_{3}\right)$ requires special attention. Suppose that $K=\mathbb{Q}(\sqrt{-3})$ and $\alpha \neq 0 \in \mathcal{O}_{K}, \operatorname{gcd}\left(\alpha, \alpha^{\tau}\right)=1$, and $N(\alpha)=p^{s}$, with $s$ an integer prime to 6 . Then the principal ideal $(\alpha)$ is equal to $(\beta)^{s}$ for some primary prime of $\mathcal{O}_{K}$ lying above $p$. There is an unique integer $\delta(\alpha)$ modulo 6 with $\alpha=\zeta_{6}^{\delta(\alpha)} \beta^{s}$. From the classical theory of cubic Gauss sums (c.f. [3], Chap. 9), one knows that $p \beta \in K\left(\boldsymbol{\mu}_{p}\right)^{\star^{3}}$. Then it follows that for any square-free odd integer $n, u=\frac{\alpha^{\tau}}{\alpha} \in K\left(\boldsymbol{\mu}_{n}\right)^{\star^{3}}$ if and only if $3 \mid \delta(\alpha)$ and $p \mid n$. In the following we shall call an imaginary quadratic integer $\alpha$ exceptional if $\alpha \in K$, and $\alpha= \pm \beta^{s}$ with $\beta$ primary prime. All other imaginary quadratic integers are called nonexceptional. From Lemma 
2.6, we know that if $\alpha$ is nonexceptional then $E_{m} \cap F_{n}=k_{m}\left(\boldsymbol{\mu}_{m}\right) \cap K\left(\boldsymbol{\mu}_{n}\right)$ always holds for relatively prime square free positive integer $m, n$ with $n$ odd.

Let $h$ denote the class number of $K$. For any positive integer $n$, let $f(n)$ denote the number of odd prime divisors of $n$. We are ready to derive a precise formula for the density.

TheOREM 3.3. Assume GRH holds. Suppose $\alpha \in \mathcal{O}_{K} \backslash \mathcal{O}_{K}{ }^{\star}, \operatorname{gcd}\left(\alpha, \alpha^{\tau}\right)=1$ and $N(\alpha)=p^{s}$, where $p$ is a prime splitting in $K, s$ is an integer satisfying $\operatorname{gcd}(6, s)=1$ and $f(s)=f\left(\frac{s}{\operatorname{gcd}(s, h)}\right)$. Then $M_{\alpha}$ has positive density given by

$$
\operatorname{den}\left(M_{\alpha}\right)= \begin{cases}\frac{1}{4} \prod_{q \mid s, q \neq p}\left(1-\frac{2}{(q-1)}\right) \prod_{q \geq 3, q \nmid p s}\left(1-\frac{2}{q(q-1)}\right) & \text { if } p \equiv 1 \text { or } \alpha \text { is exceptional } \\ \frac{1}{4} \prod_{q \mid s}\left(1-\frac{2}{(q-1)}\right) \prod_{q \geq 3, q \nmid s}\left(1-\frac{2}{q(q-1)}\right) & \text { otherwise. }\end{cases}
$$

Remark. 1. The condition $\operatorname{gcd}(6, s)=1$ in Theorem 3.3 is essential.

2. It is possible to remove the condition $f(s)=f\left(\frac{s}{\operatorname{gcd}(s, h)}\right)$ from Theorem 3.3. In doing so, one has to modify the Euler factors in the infinite product which corresponds to primes dividing $s$. Writing $(\alpha)=\mathfrak{a}^{s}$, and let $o$ be the order of the ideal class of $\mathfrak{a}$. The primes dividing $s^{\prime}=s / o$ and those dividing $o$ will give different contributions to the density, where $s^{\prime}$ is the largest integer with the property that $u \in\left(K^{\star}\right)^{s^{\prime}}$.

Proof of Theorem 3.3. Since $f(s)=f\left(\frac{s}{\operatorname{gcd}(s, h)}\right)$, one has $n_{1}=n_{2}$ for all square free integer $n$.

First Case: Suppose that $p \equiv 1(\bmod 4)$.

Case 1.1: $D_{K} \equiv 0(\bmod 4)$. we have

By Lemma 2.6 for relatively prime square free positive integers $m, n$ with $n$ odd,

$$
E_{m} \cap F_{n}= \begin{cases}\mathbb{Q}(\sqrt{p}) & \text { if } 2 \mid m \text { and } p \mid n \\ \mathbb{Q} & \text { otherwise }\end{cases}
$$

Then from Lemma 2.7 and 2.8 , we obtain

$$
c_{m, n}=1 \text { and } d_{m, n}= \begin{cases}m_{1} n_{1} \phi(m n) & \text { if } 2 p \mid m n \\ 2 m_{1} n_{1} \phi(m n) & \text { otherwise }\end{cases}
$$


Applying Theorem 3.2, we have

$$
\begin{aligned}
\operatorname{den}\left(M_{\alpha}\right) & =\sum_{\substack{m, n, 2 \nmid n \\
2 p \nmid m n}} \frac{\mu(m n)}{2 m_{1} n_{1} \phi(m n)}+\sum_{\substack{m, n, 2 \nmid n \\
2 p \mid m n}} \frac{\mu(m n)}{m_{1} n_{1} \phi(m n)} \\
& =\sum_{2 p \nmid c} \frac{2^{f(c)} \mu(c)}{2 c_{1} \phi(c)}+\sum_{2 p \mid c} \frac{2^{f(c)} \mu(c)}{c_{1} \phi(c)} \\
& =\sum_{c} \frac{2^{f(c)} \mu(c)}{2 c_{1} \phi(c)}+\sum_{2 p \mid c} \frac{2^{f(c)} \mu(c)}{2 c_{1} \phi(c)} \\
& =\frac{1}{4} \prod_{q \geq 3}\left(1-\frac{2}{q_{1}(q-1)}\right)+\frac{1}{2 p_{1}(p-1)} \prod_{q \geq 3, q \neq p}\left(1-\frac{2}{q_{1}(q-1)}\right) \\
& =\frac{1}{4} \prod_{q \geq 3, q \neq p}\left(1-\frac{2}{q_{1}(q-1)}\right) \\
& =\frac{1}{4} \prod_{q \mid s, q \neq p}\left(1-\frac{2}{(q-1)}\right) \prod_{q \geq 3, q \nmid p s}\left(1-\frac{2}{q(q-1)}\right)>0 .
\end{aligned}
$$

Case 1.2: $-D_{K} \equiv 1(\bmod 4)$, i.e. $K=\mathbb{Q}(\sqrt{-a}), a \equiv 3(\bmod 4)$. Also the integer $\alpha$ is assumed to be nonexceptional.

By Lemma 2.6, for relatively prime square free positive integers $m, n$ with $n$ odd, we have that $E_{m} \cap F_{n}=k_{m}\left(\boldsymbol{\mu}_{m}\right) \cap K\left(\boldsymbol{\mu}_{n}\right)$ is totally real except when $a \mid m n$ with $\operatorname{gcd}(a, n) \equiv 1(\bmod 4)$, in that case it contains the imaginary field $\mathbb{Q}(\sqrt{-a / \operatorname{gcd}(a, n)})$. From Lemmas 2.7 and 2.8, we get

$$
\begin{aligned}
& c_{m, n}= \begin{cases}0 & \text { if } a \mid m n \text { and } \operatorname{gcd}(a, n) \equiv 1(\bmod 4), \\
1 & \text { otherwise, }\end{cases} \\
& d_{m, n}= \begin{cases}\frac{1}{2} m_{1} n_{1} \phi(m n) & \text { if } 2 a p \mid m n, \\
m_{1} n_{1} \phi(m n) & \text { if either } a \mid m n \text { or } 2 p \mid m n, \text { but not both, } \\
2 m_{1} n_{1} \phi(m n) & \text { otherwise. }\end{cases}
\end{aligned}
$$


In order to compute $\operatorname{den}\left(M_{\alpha}\right)$ in this case, we first compute two sums $S_{1}, S_{2}$ :

$$
\begin{aligned}
S_{1} & =\sum_{\substack{m, n, 2 \nmid n \\
a \nmid m n, 2 p \nmid m n}} \frac{\mu(m n)}{2 m_{1} n_{1} \phi(m n)}+\sum_{\substack{m, n, 2 \nmid n \\
a \nmid m n, 2 p \mid m n}} \frac{\mu(m n)}{m_{1} n_{1} \phi(m n)} \\
& =\sum_{a \nmid c, 2 p \nmid c} \frac{2^{f(c)} \mu(c)}{2 c_{1} \phi(c)}+\sum_{a \nmid c, 2 p \mid c} \frac{2^{f(c)} \mu(c)}{c_{1} \phi(c)} \\
& =\sum_{a \nmid c} \frac{2^{f(c)} \mu(c)}{2 c_{1} \phi(c)}+\sum_{a \nmid c, 2 p \mid c} \frac{2^{f(c)} \mu(c)}{2 c_{1} \phi(c)} \\
& =\sum_{c} \frac{2^{f(c)} \mu(c)}{2 c_{1} \phi(c)}-\sum_{a \mid c} \frac{2^{f(c)} \mu(c)}{2 c_{1} \phi(c)}+\sum_{2 p \mid c} \frac{2^{f(c)} \mu(c)}{2 c_{1} \phi(c)}-\sum_{2 a p \mid c} \frac{2^{f(c)} \mu(c)}{2 c_{1} \phi(c)} \\
& =\frac{1}{4} \prod_{q}\left(1-\frac{2}{q_{1}(q-1)}\right)-\frac{2^{f(a)} \mu(a)}{4 a_{1} \phi(a)} \prod_{q \geq 3, q \nmid a}\left(1-\frac{2}{q_{1}(q-1)}\right) \\
& +\frac{1}{2 p_{1}(p-1)} \prod_{q \geq 3, q \neq p}\left(1-\frac{2}{q_{1}(q-1)}\right)-\frac{2^{f(a)} \mu(a)}{2 p_{1}(p-1) a_{1} \phi(a)} \prod_{q \geq 3, q \nmid a p}\left(1-\frac{2}{q_{1}(q-1)}\right) \\
& =\frac{1}{4}\left(\prod_{q \mid a}\left(1-\frac{2}{q_{1}(q-1)}\right)-\frac{2^{f(a)} \mu(a)}{a_{1} \phi(a)}\right) \prod_{q \geq 3, q \nmid a p}\left(1-\frac{2}{q_{1}(q-1)}\right) .
\end{aligned}
$$

Next consider

$$
S_{2}=\sum_{\substack{m, n, 2 \nmid n \\ a \mid m n, 2 p \nmid m n}}^{\prime} \frac{\mu(m n)}{m_{1} n_{1} \phi(m n)}+\sum_{\substack{m, n, 2 \nmid n \\ a|m n, 2 p| m n}}^{\prime} \frac{2 \mu(m n)}{m_{1} n_{1} \phi(m n)}
$$

where the dash indicates that the sum runs through $m, n$ with $\operatorname{gcd}(a, n) \equiv 3(\bmod 4)$. Define for each integer $r$ the function $f_{r}: \mathbb{Z}_{>0} \longrightarrow \mathbb{Z}$ by $f_{r}(n)=n / \operatorname{gcd}(n, r)$. Note that $n_{1}=f_{s}(n)$ for every positive integer $n$. Let $m$ and $n$ be relatively prime integer, let $a$ be a divisor of $m n$. Then one has $f_{s}(m n)=f_{s}(m) f_{s}(n), m n=a f_{a}(m) f_{a}(n)$, and

$$
f_{s}(a) \cdot f_{s}\left(f_{a}(m)\right) f_{s}\left(f_{a}(n)\right)=f_{s}\left(a f_{a}(m) f_{a}(n)\right)=f_{s}(m n)=f_{s}(m) f_{s}(n)
$$

Thus

$$
\frac{\mu(m n)}{m_{1} n_{1} \phi(m n)}=\frac{\mu(a)}{a_{1} \phi(a)} \frac{\mu\left(f_{a}(m) f_{a}(n)\right)}{f_{a}(m)_{1} f_{a}(n)_{1} \phi\left(f_{a}(m) f_{a}(n)\right)} .
$$

For any positive integer $n$, let $g(n)$ denote the number of prime divisors of $n$ that is congruent to 3 modulo 4 . For each ordered-pair $\left(m^{\prime}, n^{\prime}\right)$ with $\operatorname{gcd}\left(a, m^{\prime} n^{\prime}\right)=1$, the number of ordered pair $(m, n)$ with $a \mid m n, n$ odd, and $g(\operatorname{gcd}(a, n))$ odd satisfying $m^{\prime}=f_{a}(m)$ and $n^{\prime}=f_{a}(n)$ is equal to

$$
\begin{aligned}
& \left(\left(\begin{array}{c}
g(a) \\
1
\end{array}\right)+\left(\begin{array}{c}
g(a) \\
3
\end{array}\right)+\cdots\left(\begin{array}{c}
g(a) \\
g(a)
\end{array}\right)\right) \cdot 2^{f(a)-g(a)} \\
= & 2^{g(a)-1} \cdot 2^{f(a)-g(a)}=2^{f(a)-1} .
\end{aligned}
$$


Therefore,

$$
\begin{aligned}
S_{2} & =\frac{2^{f(a)-1} \mu(a)}{a_{1} \phi(a)}\left(\sum_{\substack{m, n, 2 \nmid n \\
2 p \nmid m n \operatorname{gcd}(a, m n)=1}} \frac{\mu(m n)}{m_{1} n_{1} \phi(m n)}+\sum_{\substack{m, n, 2 \nmid n \\
\operatorname{gcd}(a, m n)=1,2 p \mid m n}} \frac{2 \mu(m n)}{m_{1} n_{1} \phi(m n)}\right) \\
& =\frac{2^{f(a)} \mu(a)}{2 a_{1} \phi(a)}\left(\sum_{\operatorname{gcd}(a, c)=1,2 p \nmid c} \frac{2^{f(c)} \mu(c)}{c_{1} \phi(c)}+\sum_{\operatorname{gcd}(a, c)=1,2 p \mid c} \frac{2^{f(c)+1} \mu(c)}{c_{1} \phi(c)}\right) \\
& =\frac{2^{f(a)} \mu(a)}{2 a_{1} \phi(a)}\left(\sum_{\operatorname{gcd}(a, c)=1} \frac{2^{f(c)} \mu(c)}{c_{1} \phi(c)}+\sum_{\operatorname{gcd}(a, c)=1,2 p \mid c} \frac{2^{f(c)} \mu(c)}{c_{1} \phi(c)}\right) \\
& =\frac{2^{f(a)} \mu(a)}{4 a_{1} \phi(a)} \prod_{q \geq 3, q \nmid a}\left(1-\frac{2}{q_{1}(q-1)}\right)+\frac{2^{f(a)} \mu(a)}{2 p_{1}(p-1) a_{1} \phi(a)} \prod_{q \geq 3, q \nmid a p}\left(1-\frac{2}{q_{1}(q-1)}\right) \\
& =\frac{2^{f(a)} \mu(a)}{4 a_{1} \phi(a)} \prod_{q \geq 3, q \nmid a p}\left(1-\frac{2}{q_{1}(q-1)}\right) .
\end{aligned}
$$

Applying Theorem 3.2, we have

$$
\operatorname{den}\left(M_{\alpha}\right)=S_{1}+S_{2}=\frac{1}{4} \prod_{q \geq 3, q \neq p}\left(1-\frac{2}{q_{1}(q-1)}\right)
$$

Case 1.3: Suppose $K=\mathbb{Q}(\sqrt{-3})$ and $\alpha$ is exceptional

For square free $m, n, n$ odd and $3 \nmid m$, we compute $\left[E_{m} \cap F_{n}: \mathbb{Q}\right]$ using Lemma 2.6. If $\operatorname{gcd}(3 p, n)=1$ and $2 \nmid m$, we obtain $E_{m} \cap F_{n}=\mathbb{Q}$. If $p \mid m$ and $3 \mid n$, then as in Case 3.1 $E_{m} \cap F_{n}=k_{m}\left(\boldsymbol{\mu}_{m}\right) \cap K\left(\boldsymbol{\mu}_{n}, \sqrt[3]{u}\right)$ is cubic over $\mathbb{Q}$ no matter $m$ is even or odd. When $m$ is even, we also have $E_{m} \cap F_{n}=\mathbb{Q}$ if $\operatorname{gcd}(3 p, n)=1$ or $p \nmid m n$. On the other hand, $E_{m} \cap F_{n}=\mathbb{Q}(\sqrt{p})$ if $p \mid n$. Therefore $E_{m} \cap F_{n}$ is always totally real, and

$$
c_{m, n}= \begin{cases}0 & \text { if } 3 \mid m, \\ 1 & \text { if } 3 \nmid m .\end{cases}
$$

By Lemma 2.8, If $3 \nmid m$ and $2 \nmid m$, then

$$
d_{m, n}= \begin{cases}2 m_{1} n_{1} \phi(m n) & \text { if } 3 \nmid n, \\ m_{1} n_{1} \phi(m n) & \text { if } 3 \mid n \text { and } p \nmid m n \\ \frac{m_{1} n_{1} \phi(m n)}{3} & \text { if } 3 \mid n \text { and } p \mid m n\end{cases}
$$

If $3 \nmid m$ and $2 \mid m$, then

$$
d_{m, n}= \begin{cases}2 m_{1} n_{1} \phi(m n) & \text { if } 3 \nmid n \text { and } p \nmid m n, \\ m_{1} n_{1} \phi(m n) & \text { if } 3 \nmid n \text { and } p \mid m n, \\ m_{1} n_{1} \phi(m n) & \text { if } 3 \mid n \text { and } p \nmid m n, \\ \frac{m_{1} n_{1} \phi(m n)}{6} & \text { if } 3 \mid n \text { and } p \mid m n .\end{cases}
$$


In order to compute $\operatorname{den}\left(M_{\alpha}\right)$, we first evaluate sums $S_{3}, S_{4}$ as follows:

$$
\begin{aligned}
& S_{3}=\sum_{\substack{m, n, 2 \nmid n \\
3 \nmid m n, 2 \nmid m}} \frac{\mu(m n)}{2 m_{1} n_{1} \phi(m n)}+\sum_{\substack{m, n, 2 \nmid n \\
3 \nmid m n, 2 \mid m \\
p \nmid m n}} \frac{\mu(m n)}{2 m_{1} n_{1} \phi(m n)}+\sum_{\substack{m, n, 2 \nmid n \\
3 \nmid m n, 2|m \\
p| m n}} \frac{\mu(m n)}{m_{1} n_{1} \phi(m n)} \\
& =\sum_{\substack{m, n, 2 \nmid n \\
3 \nmid m n}} \frac{\mu(m n)}{2 m_{1} n_{1} \phi(m n)}+\sum_{\substack{m, n, 2 \nmid n \\
3 \nmid m n, 2|m, p| m n}} \frac{\mu(m n)}{2 m_{1} n_{1} \phi(m n)} \\
& =\frac{1}{4} \sum_{\substack{m, n \\
\operatorname{gcd}(6, m n)=1}} \frac{\mu(m n)}{m_{1} n_{1} \phi(m n)}-\frac{1}{4} \sum_{\substack{m, n \\
\operatorname{gcd}(6, m n)=1, p \mid m n}} \frac{\mu(m n)}{m_{1} n_{1} \phi(m n)} \\
& =\frac{1}{4} \sum_{\operatorname{gcd}(6, c)=1} \frac{2^{f(c)} \mu(c)}{c_{1} \phi(c)}-\frac{1}{4} \sum_{\operatorname{gcd}(6, c)=1, p \mid c} \frac{2^{f(c)} \mu(c)}{c_{1} \phi(c)}=\frac{1}{4} \sum_{\operatorname{gcd}(6 p, c)=1} \frac{2^{f(c)} \mu(c)}{c_{1} \phi(c)}, \\
& S_{4}=\sum_{\substack{m, n, 2 \nmid n \\
3 \nmid m, 2 \nmid m, 3|n, p| m n}} \frac{3 \mu(m n)}{m_{1} n_{1} \phi(m n)}+\sum_{\substack{m, n, 2 \nmid n \\
3 \nmid m, 2|m, 3| n, p \mid m n}} \frac{6 \mu(m n)}{m_{1} n_{1} \phi(m n)}=0 .
\end{aligned}
$$

Applying Theorem 3.2, we have

$$
\begin{aligned}
& \operatorname{den}\left(M_{\alpha}\right)=S_{3}+S_{4}+\sum_{\substack{m, n, 2 \nmid n \\
3 \nmid m, 3 \mid n, p \nmid m n}} \frac{\mu(m n)}{m_{1} n_{1} \phi(m n)} \\
& =\frac{1}{4} \sum_{\operatorname{gcd}(6 p, c)=1} \frac{2^{f(c)} \mu(c)}{c_{1} \phi(c)}-\frac{1}{12} \sum_{\operatorname{gcd}(6 p, c)=1} \frac{2^{f(c)} \mu(c)}{c_{1} \phi(c)} \\
& =\frac{1}{6} \sum_{\operatorname{gcd}(6 p, c)=1} \frac{2^{f(c)} \mu(c)}{c_{1} \phi(c)}=\frac{1}{4} \prod_{q \mid s, q \neq p}\left(1-\frac{2}{(q-1)}\right) \prod_{q \geq 3, q \nmid p s}\left(1-\frac{2}{q(q-1)}\right) .
\end{aligned}
$$

Second Case: Suppose $p \not \equiv 1(\bmod 4)$.

Case 2.1: $D_{K} \equiv 0(\bmod 4)$. Then the possibility is $D_{K} \equiv 4(\bmod 8)$ and $p \equiv 3$ $(\bmod 4)$.

By Lemmas 2.6, 2.7 and 2.8, for relatively prime square free positive integers $m, n$ with $n$ odd, we have

$$
E_{m} \cap F_{n}=\mathbb{Q}, c_{m, n}=1, \text { and } d_{m, n}=2 m_{1} n_{1} \phi(m n) .
$$

Then by Theorem 3.2 , we have

$$
\begin{aligned}
\operatorname{den}\left(M_{\alpha}\right) & =\sum_{m, n, 2 \nmid n} \frac{\mu(m n)}{2 m_{1} n_{1} \phi(m n)}=\sum_{c} \frac{2^{f(c)} \mu(c)}{2 c_{1} \phi(c)} \\
& =\frac{1}{4} \prod_{q \geq 3}\left(1-\frac{2}{q_{1}(q-1)}\right) \\
& =\frac{1}{4} \prod_{q \mid s}\left(1-\frac{2}{(q-1)}\right) \prod_{q \geq 3, q \nmid s}\left(1-\frac{2}{q(q-1)}\right)>0 .
\end{aligned}
$$


Case 2.2: $-D_{K} \equiv 1(\bmod 4)$, i.e. $a \equiv 3(\bmod 4)$. The integer $\alpha$ is assumed to be nonexceptional. Note the case $p=2$ is allowed here.

By Lemmas 2.6, 2.7 and 2.8, for relatively prime square free positive integers $m, n$ with $n$ odd, we have

$$
\begin{aligned}
E_{m} \cap F_{n} & = \begin{cases}\mathbb{Q}(\sqrt{-a / \operatorname{gcd}(a, n)}) & \text { if } a \mid m n \text { and } \operatorname{gcd}(a, n) \equiv 1(\bmod 4), \\
\mathbb{Q}(\sqrt{a / \operatorname{gcd}(a, n)}) & \text { if } a \mid m n \text { and } \operatorname{gcd}(a, n) \equiv 3(\bmod 4), \\
\mathbb{Q} & \text { otherwise, }\end{cases} \\
c_{m, n} & = \begin{cases}0 & \text { if } a \mid m n \text { and } \operatorname{gcd}(a, n) \equiv 1(\bmod 4), \\
1 & \text { otherwise, }\end{cases} \\
d_{m, n} & = \begin{cases}m_{1} n_{1} \phi(m n) & \text { if } a \mid m n, \\
2 m_{1} n_{1} \phi(m n) & \text { otherwise. }\end{cases}
\end{aligned}
$$

We start with the sum

$$
S_{5}=\sum_{\substack{m, n, 2 \nmid n \\ a \mid m n}}^{\prime} \frac{\mu(m n)}{m_{1} n_{1} \phi(m n)}
$$

where the dash indicates that the sum runs through $m, n$ with $\operatorname{gcd}(a, n) \equiv 3(\bmod 4)$. Similar to the computation of $S_{2}$, we have

$$
S_{5}=\frac{2^{f(a)} \mu(a)}{2 a_{1} \phi(a)} \sum_{\operatorname{gcd}(a, c)=1} \frac{2^{f(c)} \mu(c)}{c_{1} \phi(c)} .
$$

Applying Theorem 3.2, we have

$$
\begin{aligned}
\operatorname{den}\left(M_{\alpha}\right) & =\sum_{\substack{m, n, 2 \nmid n \\
a \nmid m n}} \frac{\mu(m n)}{2 m_{1} n_{1} \phi(m n)}+S_{5} \\
& =\sum_{c} \frac{2^{f(c)} \mu(c)}{2 c_{1} \phi(c)}=\frac{1}{4} \prod_{q \geq 3}\left(1-\frac{2}{q_{1}(q-1)}\right) .
\end{aligned}
$$

Case 2.3: Suppose $K=\mathbb{Q}(\sqrt{-3})$ and $\alpha$ is exceptional. Note that $p \equiv 3 \bmod 4$.

Using Lemma 2.6, for square free $m, n, n$ odd and $3 \nmid m$, we compute $E_{m} \cap F_{n}=\mathbb{Q}$ if $3 \nmid n$, or $p \nmid n$. On the other hand, if $p \mid m$ and $3 \mid n$, then $E_{m} \cap F_{n}=k_{m}\left(\boldsymbol{\mu}_{m}\right) \cap$ $K\left(\boldsymbol{\mu}_{n}, \sqrt[3]{u}\right)$ is a cubic extension of $\mathbb{Q}$. Thus $E_{m} \cap F_{n}$ is always totally real, and

$$
c_{m, n}= \begin{cases}0 & \text { if } 3 \mid m, \\ 1 & \text { if } 3 \nmid m .\end{cases}
$$

From Lemma 2.8, we also obtain, for $3 \nmid m$ :

$$
d_{m, n}= \begin{cases}2 m_{1} n_{1} \phi(m n) & \text { if } 3 \nmid n, \\ m_{1} n_{1} \phi(m n) & \text { if } 3 \mid n \text { and } p \nmid m n, \\ \frac{m_{1} n_{1} \phi(m n)}{3} & \text { if } 3 \mid n \text { and } p \mid m n .\end{cases}
$$


Applying Theorem 3.2, we see that the value of $\operatorname{den}\left(M_{\alpha}\right)$ is

$$
\begin{aligned}
& \sum_{\substack{m, n, 2 \nmid n \\
3 \nmid m n}} \frac{\mu(m n)}{2 m_{1} n_{1} \phi(m n)}+\sum_{\substack{m, n, 2 \nmid n \\
3 \nmid m, 3 \mid n, p \nmid m n}} \frac{\mu(m n)}{m_{1} n_{1} \phi(m n)}+\sum_{\substack{m, n \\
\operatorname{cod}(6, m, 2 \nmid n \\
3 \nmid m, 3|n, p| m n}} \frac{3 \mu(m n)}{m_{1} n_{1} \phi(m n)} \\
= & \sum_{\substack{m(m n) \\
\operatorname{gcd}(6, m n)=1}}-\sum_{\substack{m, n \\
\operatorname{gcd}(6 p, m n)=1}} \frac{\mu(m n)}{12 m_{1} n_{1} \phi(m n)} \\
& -\sum_{\substack{m, n \\
\operatorname{gcd}(6 p, m n)=p}} \frac{\mu(m n)}{4 m_{1} n_{1} \phi(m n)} \\
= & \frac{1}{4} \sum_{\operatorname{gcd}(6, c)=1} \frac{2^{f(c)} \mu(c)}{c_{1} \phi(c)}-\frac{1}{12} \sum_{\operatorname{gcd}(6 p, c)=1} \frac{2^{f(c)} \mu(c)}{c_{1} \phi(c)}-\frac{1}{4} \sum_{\operatorname{gcd}(6, c)=1, p \mid c} \frac{2^{f(c)} \mu(c)}{c_{1} \phi(c)} \\
= & \frac{1}{6} \sum_{\operatorname{gcd}(6 p, c)=1} \frac{2^{f(c)} \mu(c)}{c_{1} \phi(c)}=\frac{1}{4} \prod_{q \mid s, q \neq p}\left(1-\frac{2}{(q-1)}\right) \prod_{q \geq 3, q \nmid p s}\left(1-\frac{2}{q(q-1)}\right) .
\end{aligned}
$$

4. Applications to Elliptic Curves over Finite Fields. Let $\mathbb{F}_{r}$ denote a finite field of characteristic $p$ with $r=p^{s}$ elements. Given an elliptic curve $E$ defined over $\mathbb{F}_{r}$, we would like to know the size of the Galois extension of $\mathbb{F}_{r}$ obtained through adjoining coordinates of all $\ell$-torsion points where $\ell$ is a prime. Let $E[\ell] \subset E\left(\mathbb{F}_{r}\right)$ be the set of all these $\ell$-torsion points. Let End $_{E}$ denote the endomorphism ring of $E$ and let $\alpha=\alpha_{E} \in \operatorname{End}_{E}$ be the Frobenius endomorphism which raises the coordinates of points on $E$ to its $r$-th power. Then the size of the Galois extension in question is the degree $\left[\mathbb{F}_{r}(E[\ell]): \mathbb{F}_{r}\right]$ which equals to the order of the Frobenius endomorphism acting on $E[\ell]$. If the curve $E$ is not supersingular, a well-known theorem of Hasse asserts that $\mathbb{Z}[\alpha] \subset \operatorname{End}_{E}$ which can be identified with an order in an imaginary quadratic field $K=K_{E}$. If $E$ is supersingular, it may happen that $\alpha_{E} \in \mathbb{Z}$, or else $\mathbb{Z}[\alpha]$ is still contained in an imaginary quadratic field $K=K_{E}$. We let $\operatorname{disc}(\alpha)$ be the discriminant of $\mathbb{Z}[\alpha]$. The following proposition bounds $\left[\mathbb{F}_{r}(E[\ell]): \mathbb{F}_{r}\right]$ in the non-supersingular case:

Proposition 4.1. Given a non-supersingular elliptic curve $E_{/ \mathbb{F}_{r}}$ with (geometric) Frobenius endomorphism $\alpha$ embedded in an imaginary quadratic field $K$. Let $e_{2}$ be the largest divisor of 24 such that $\alpha \in\left(K^{\star}\right)^{e_{2}}$, and $e_{1}=2$, or 1 according to whether $\alpha$ is a square in $K$. Suppose prime $\ell>3$ and $\ell \nmid p \operatorname{disc}(\alpha)$. Then

$$
\left[\mathbb{F}_{r}(E[\ell]): \mathbb{F}_{r}\right] \leq \begin{cases}\frac{\ell^{2}-1}{e_{2}}, & \text { if } \ell \text { is inert in } K / \mathbb{Q} \\ \frac{\ell-1}{e_{1}}, & \text { if } \ell \text { splits in } K / \mathbb{Q}\end{cases}
$$

Proof. The degree $\left[\mathbb{F}_{r}(E[\ell]): \mathbb{F}_{r}\right]$ is exactly the order of the endomorphism $\alpha$ inside $\left(\operatorname{End}_{E} / \ell \operatorname{End}_{E}\right)^{\star}$. Since $\ell$ does not divide $\operatorname{disc}(\alpha)$, we have $\mathbb{Z}[\alpha] / \ell \mathbb{Z}[\alpha] \cong$ $\operatorname{End}_{E} / \ell \operatorname{End}_{E} \cong \mathcal{O}_{K} / \ell \mathcal{O}_{K}$, hence $\left[\mathbb{F}_{r}(E[\ell]): \mathbb{F}_{r}\right]$ equals the order of $\alpha$ inside the group $\left(\mathcal{O}_{K} / \ell \mathcal{O}_{K}\right)^{\star}$. If $\ell$ is inert in $K / \mathbb{Q}$, then we have $\#\left(\left(\mathcal{O}_{K} / \ell \mathcal{O}_{K}\right)^{\star}\right)=\ell^{2}-1$ which is divisible by 24 . On the other hand if $\ell$ splits in $K / \mathbb{Q}$, the group $\left(\mathcal{O}_{K} / \ell \mathcal{O}_{K}\right)^{\star}$ has exponent $\ell-1$. The desired bound follows immediately from these observations.

We are interested in the distribution of the degrees $\left[\mathbb{F}_{r}(E[\ell]): \mathbb{F}_{r}\right]$ as the prime number $\ell$ varies. In particular, how often the Galois extension degree $\left[\mathbb{F}_{r}(E[\ell]): \mathbb{F}_{r}\right]$ can be the largest possible, in other words, is equal to $\left(\ell^{2}-1\right) / e_{2}$ ? We consider 
therefore the following set of primes:

$$
M_{E}=\left\{\ell \mid \ell \in \mathbb{P},\left[\mathbb{F}_{r}(E[\ell]): \mathbb{F}_{r}\right]=\left(\ell^{2}-1\right) / e_{2}\right\}
$$

The main theorem to be established is:

THEOREM 4.2. Assume GRH holds, and suppose $\operatorname{gcd}(s, 6)=1$. Let $E_{/ \mathbb{F}_{r}}$ be any elliptic curve which is not supersingular. Then the set $M_{E}$ always has positive density.

Proof. Let $K=K_{E}$, with $h$ equals to the class number of $\mathcal{O}_{K}$. First, we apply Theorem 3.2 to the Frobenius $\alpha=\alpha_{E}$. This shows that the set $M_{E}$ has a density, since it differs from $M_{\alpha}$ only by a finite set. Next we can multiply $s$ by suitable powers of those prime factors of $h$ not dividing 6 so that $s^{\prime}$ and $s^{\prime} / \operatorname{gcd}\left(s^{\prime}, h\right)$ has the same set of odd prime factors. Extending the base field to $\mathbb{F}_{p^{s^{\prime}}}$, and replacing the curve $E$ by $E^{\prime}$ which is the original $E$ over $\mathbb{F}_{p^{s^{\prime}}}$. Then the Frobenius $\alpha^{\prime}=\alpha_{E^{\prime}}$ satisfies the hypothesis of Theorem 3.3. It follows that the set $M_{E^{\prime}}$ has positive density. To finish the proof, it suffices to show that $M_{\alpha^{\prime}} \subseteq M_{\alpha}$. This follows from the fact that the order of $\alpha$ modulo $\ell$ is at least the order of $\alpha^{\prime}$ modulo $\ell$ because $\alpha^{\prime}$ is a power of $\alpha$.

For prime fields $\mathbb{F}_{r}=\mathbb{F}_{p}$, a precise value of the density can be given.

THEOREM 4.3. Given an elliptic curve $E_{/ \mathbb{F}_{p}}$ which is not supersingular. Suppose GRH holds. Then the density of $M_{E}$ is :

$$
\operatorname{den}\left(M_{E}\right)= \begin{cases}\left(1-\frac{2}{p(p-1)}\right)^{-1} C_{2} & \text { if } p \equiv 1(\bmod 4) \text { or } \alpha \text { is exceptional, } \\ C_{2} & \text { otherwise, }\end{cases}
$$

where $C_{2}$ is the constant:

$$
C_{2}=\frac{1}{4} \prod_{q \neq 2}\left(1-\frac{2}{q(q-1)}\right)=0.133776 \cdots
$$

Proof. Since $\operatorname{den}\left(M_{E}\right)=\operatorname{den}\left(M_{\alpha}\right)$ in this case ( $\left.\mathrm{s}=1\right)$, the formula follows from Theorem 3.3 immediately.

Let $t_{E} \in \mathbb{Z}$ denote the trace of the Frobenius endomorphism. If the curve $E$ is supersingular, bounds on $\left[\mathbb{F}_{r}(E[\ell]): \mathbb{F}_{r}\right]$ are given by Then

Proposition 4.4. Suppose $E_{/ \mathbb{F}_{r}}$ is supersingular and $\ell$ does not divide $\operatorname{disc}(\alpha)$.

$$
\left[\mathbb{F}_{r}(E[\ell]): \mathbb{F}_{r}\right] \leq \begin{cases}(\ell-1), & \text { if } t_{E}= \pm 2 \sqrt{r}, \text { and } s \text { even } \\ 2(\ell-1), & \text { if } t_{E}=0 \\ 3(\ell-1), & \text { if } t_{E}= \pm \sqrt{r}, \text { and } s \text { even } \\ 4(\ell-1), & \text { if } t_{E}= \pm p^{(s+1) / 2}, s \text { odd, and } p=2 \\ 6(\ell-1), & \text { if } t_{E}= \pm p^{(s+1) / 2}, s \text { odd, and } p=3\end{cases}
$$

Proof. Frobenius endomorphisms of all supersingular elliptic curves have been computed explicitly by Deuring (c.f.[7], Theorem 4.1). If $t_{E}= \pm 2 \sqrt{r}$ and $s$ is even, $\alpha_{E} \in \mathbb{Z}$. If $t_{E}=0, \alpha_{E}= \pm \sqrt{-r}$, then $\alpha_{E}^{2} \in \mathbb{Z}$. If $t_{E}= \pm \sqrt{r}$, and $s$ is even, $\alpha_{E}= \pm p^{\frac{s}{2}} \frac{1 \pm \sqrt{-3}}{2}, \alpha_{E}^{3} \in \mathbb{Z}$. If $t_{E}= \pm 2^{\frac{(s+1)}{2}}$ and $s$ is odd, $\alpha_{E}= \pm 2^{\frac{s+1}{2}}(1 \pm \sqrt{-1})$, $\alpha_{E}^{4} \in \mathbb{Z}$. If $t_{E}= \pm 3^{\frac{(s+1)}{2}}$ and $s$ is odd, $\alpha_{E}= \pm 3^{\frac{s-1}{2}} \frac{3 \pm \sqrt{-3}}{2}, \alpha_{E}^{6} \in \mathbb{Z}$. The proposition follows from this information immediately.

Combining Theorem 4.3 with Proposition 4.4 we obtain the following characterization of supersingular elliptic curves:

COROLlary 4.5. Assume GRH holds. Then $E_{/ \mathbb{F}_{p}}$ is supersingular if and only if $\left[\mathbb{F}_{p}(E[\ell]): \mathbb{F}_{p}\right]=O(\ell-1)$ as $\ell$ runs through the rational primes. 
In fact, in the supersingular case, it is not difficult to derive from Hooley's classical work on Artin's primitive roots conjecture (c.f. [2] or [4], the details are left to the reader) for the torus $\mathbb{G}_{m}$, the following result

THEOREM 4.6. Assume $G R H$ holds. Let $E_{/ \mathbb{F}_{p}}$ be a supersingular elliptic curve. Then the set of primes $\ell$ satisfying $\left[\mathbb{F}_{p}(E[\ell]): \mathbb{F}_{p}\right]=2(\ell-1)$ has a positive density.

\section{REFERENCES}

[1] Y.-M. J. CHEN, On primitive roots of one-dimensional tori, Preprint, 2000.

[2] C. Hooley, On Artin's conjecture, J. reine angew Math., 225 (1967), pp. 209-220.

[3] K. Ireland and M. Rosen, A Classical Introduction to Modern Number Theory, SpringerVerlag, New York, 1982.

[4] M. R. MurTy, On Artin's conjecture, Journal of Number Theory, 16 (1983), pp. 147-168.

[5] G. RotA, On the foundations of combinatorial theory, I. theory of möbius functions, Z. Wahrsch. Verw. Gebiete, 2 (1964), pp. 340-368.

[6] J.-P. Serre, Quelques applications du Théorème de densité de Chebotarev, Publ. Math. IHES, 54 (1981), pp. 123-201.

[7] W. C. Waterhouse, Abelian varieties over finite fields, Ann. scient. EC. Norm. Sup., 2 (1969), pp. 521-560. 
